\title{
Matching Theory as Enabler of Efficient Spectrum Management in 5G Networks
}

\author{
Christos Tsirakis ${ }^{1}$, Elena Lopez-Aguilera ${ }^{2}$, George Agapiou ${ }^{3}$, Dimitris Varoutas ${ }^{4}$ \\ ${ }^{1}$ OTE Academy S.A., Department of Informatics and Telecommunications, National and Kapodistrian University of \\ Athens, Athens, Greece, ctsirakis@oteacademy.gr, ctsirakis@di.uoa.gr \\ ${ }^{2}$ Department of Network Engineering, Universitat Politècnica de Catalunya, Barcelona, Spain, elopez@entel.upc.edu \\ ${ }^{3}$ OTE S.A., Athens, Greece, gagapiou@oteresearch.gr \\ ${ }^{4}$ Department of Informatics and Telecommunications, National and Kapodistrian University of Athens, Athens,
} Greece,D.Varoutas@di.uoa.gr

\begin{abstract}
This paper analyzes the spectrum trading problem in virtualized fifth generation (5G) networks in order to enhance the network performance with respect to the spectrum utilization. The problem is modeled as a Many-to-Many Matching (M2MM) game with utility-based preferences and determines the matching between mobile network operators and mobile virtual network operators. The two proposed versions of utility functions for each set aim at maximizing the satisfaction of both sets with conflicting interests and improving the overall spectrum efficiency. In the simulation evaluation, the proposed scheme is compared with three different schemes in terms of the system utility, individual and pair matching satisfaction. We also investigate the scalability aspects, the strategy plan impact on the matching performance of our proposed scheme, and, at the same time, we attempt to make appropriate assumptions closer to reality. Our proposed scheme shows much better performance than the other schemes achieving a quite high level of satisfaction for the matching result on both sets.
\end{abstract}

Keywords—spectrum trading, 5G, virtualization, matching theory, many-to-many.

\section{INTRODUCTION}

Undoubtedly, the most precious and scarce resource for wireless communications is spectrum frequency. Currently, the spectrum policy determines that most of the available spectrum is assigned to wireless mobile companies on a long term basis for large geographical regions. ${ }^{1}$ Additionally, many mobile subscribers, several wireless devices per user and thousands of new applications create the need for frequency bands. As a result, both licensed and unlicensed bands are becoming congested and several times licensed bands are identified as underutilized, ${ }^{2}$ even in the most overcrowded cities, such as New York, ${ }^{3}$ due to spectrum mismanagement. Therefore, this issue quickly leads to the investigation of solutions for reliable utilization of licensed spectrum bands.

Besides, the forthcoming $5 \mathrm{G}$ networks are anticipated to bring not only very high throughput, exceptional quality of user experience, and ultra-low latency, but also improved spectral efficiency, compared to the current legacy technologies of $3 \mathrm{G}$ and $4 \mathrm{G}$ networks. In general, addressing these requirements will demand much bigger bandwidth, densification of small cells and wide use of massive MIMO and millimeter-wave technologies. ${ }^{4}$ More specifically, the densification of small cells will increase both the network capacity and spectral efficiency by offloading traffic from macro cells and by reducing the cell radius, respectively. ${ }^{5}$ Then, the millimeter-wave frequency bands will increase the throughput by offering wide chunks of available unused bandwidth and their limited propagation characteristics (i.e., high path loss) can fit well with the densification of small cells. In the same sense, massive MIMO technology will not only increase the throughput, but it will also improve the spectral efficiency by applying spatial multiplexing of many terminals in the same time-frequency resource. ${ }^{6}$

In fact, $5 \mathrm{G}$ technology and its attributes can improve the spectral efficiency. Additionally, wireless virtualization, as a new fundamental functionality for the $5 \mathrm{G}$ networks, ${ }^{7}$ could help the efficient management of spectrum resources, too. Resource virtualization helps simplify the control of virtual networks and avoid overprovisioning by assigning wireless resources intelligently based on the actual need. ${ }^{8}$ Essentially, wireless virtualization concerns the creation of multiple separate virtual networks by physical resource sharing. This major concept relies on two 5G key enablers, Software Defined Network (SDN) that enables the decoupling of the 
infrastructure from the offered services, and Network Function Virtualization (NFV) that refers to the implementation of network functions in software running on general-purpose computing/storage platforms. In this case, the wireless virtualized business model mainly includes the Mobile Network Operators (MNOs) that provide their own infrastructure and/or radio resources to the Mobile Virtual Network Operators (MVNOs). ${ }^{9}$ Specifically, the physical resources (e.g., infrastructure, spectrum) of a base station owned by an MNO are abstracted into isolated virtual resources, which are then leased and transparently shared among various MVNOs. So, it becomes clear that virtualization can reduce the capital expenditure (CapEx) and operational expenditure $(\mathrm{OpEx})$ by minimizing the cost spent for new deployments and maintenance. Also, virtualization can achieve considerable improvement at resource utilization and quality of service, by efficiently meeting the dynamic service requirements triggered by the customers of different MVNOs.

Several spectrum sharing schemes have been recommended to enhance the spectrum efficiency by allowing utilization of unused or underutilized spectrum temporally and geographically. ${ }^{10}$ However, these schemes actually involve not only spectrum sharing but also economic transactions between various parties, thus introducing spectrum trading. By definition, spectrum trading is the mechanism of buying and selling the rights of spectrum frequencies. ${ }^{11}$ The crucial benefits of spectrum trading are that the spectrum owners can make more money by selling their unutilized spectrum frequencies. Also, spectrum leasers can gain temporal but guaranteed spectrum access to serve their particular needs. ${ }^{1}$ It actually gives them the opportunity to provide wireless services in a better way and develop economically, and creates new business opportunities. In fact, over the past years, the traditional market dominated by MNOs with dedicated spectrum has left its place to the spectrum trading market, where MVNOs have become strong players by utilizing unused spectrum. Thus, they get the chance to bring their own featured services, support their traffic demands, and finally attract greater number of customers. ${ }^{9}$

However, the parties involved (i.e., MNOs and MVNOs) are self-interested. In fact, both parties act selfishly, MNOs want to gain more revenues for certain bandwidth, MVNOs want to gain more bandwidth with certain cost, but they finally proceed in exchanging this kind of goods. As a result, spectrum trading can generate both competition and cooperation between buyers and sellers. Thus, a significant challenge for virtualized $5 \mathrm{G}$ networks is the spectrum trading and how to efficiently allocate the available spectrum resources in order both MNOs and MVNOs to be satisfied. ${ }^{12}$

This paper concentrates on the topic of spectrum management and trading in virtualized 5G networks and how matching theory enables us to resolve this. The related work is thoroughly studied in Section II. Then, in Section III, the virtualized network model in the spectrum trading market along with the proposed matching algorithm are defined. Also, in Section IV and Section V, the simulation results of the matching performance at various different scenarios are presented for the proposed and enhanced version of the utility functions, respectively. Finally, in Section VI, the conclusions of our paper are demonstrated and some possible future work is suggested.

\section{RELATED WORK}

The resource allocation problem in virtualized networks, i.e., how to assign resources to different slices, ${ }^{13}$ has been extensively studied and various solutions have been proposed. VMWare, for instance, has developed a network virtualization platform (NVP) ${ }^{14}$ as a complete network virtualization solution that allows the creation of independent virtual networks for multi-tenant networks. Spectrum virtualization can be implemented by sharing the RF front end and antenna of the base station, ${ }^{15}$ where the radio is flexibly sliced into multiple slices, each operating on different frequencies. However, the most usual approach to enable spectrum virtualization is to let each MVNO have its own customized scheduler over its slice. Then, the frame scheduler's modification is done in order to assign Physical Resource Blocks (PRBs) to the slices in a dynamic manner at equal time intervals. ${ }^{16,17,18}$ Thus, a hypervisor is added on the top of the physical layer of the base station and is responsible for virtualizing the base station and the spectrum as well. In this way, each MVNO will only get its required share of the PRBs and less waste of resources will occur. ${ }^{19}$ Also, another approach ${ }^{20}$ adopts a high-level strategy by proposing a mechanism that schedules the resources between slices at the MAC layer or Network layer. In this work, the authors define the Network Virtualization Substrate (NVS), which is a substrate on BSs, by integrating virtualization into the WiMAX base station uplink/downlink scheduler software.

However, when economic transactions take place on top of the spectrum allocation scenarios, then spectrum trading dominates, as described in Section I. Presently, different approaches have been adopted to cope with the spectrum trading problem, like game theory, auction theory, and market equilibrium. ${ }^{21}$ 
Game theory includes competition or cooperation among multiple sellers and buyers to reach Nash equilibrium solution, which optimizes the payoff of all the players. On this point, a bankruptcy game was suggested for dynamic wireless resource allocation among multiple operators. ${ }^{22}$ Nevertheless, not all solutions converge to an equilibrium. Moreover, when huge number of players are involved, communication overhead can be a critical issue in game theory. Also, the high computational complexity of game theory, can be one more significant flaw. ${ }^{1}$

Auction is the most well-known approach for trading in real world markets. In an auction, buyers submit their bids to the seller and the spectrum is sold to the buyer with the highest bid. In this way, the seller maximizes its profit and the auction ends. On this matter, an auction-based spectrum trading approach is applied to maximize both the total buyer's satisfaction and the seller's profit. ${ }^{23}$ Specifically, the shared used model and the risk of imperfect spectrum sensing are considered in order to derive an expression that optimizes the sensing time (i.e., the time that the base station needs in order to identify spectrum opportunities, thus avoiding to make interferences with other base stations). Also, a hierarchical auction mechanism composed of two hierarchical auction models (i.e., a single-seller multiplebuyer model and an extended multiple-seller multiplebuyer model) has been designed. ${ }^{12}$ However, auctions may not be appropriate for dynamic and short-term frequency assignment, since they may require many repetitions to reach a stable solution. ${ }^{1}$

Market equilibrium is the condition when the supply of a product is equal to the demand of this product. By relying only on supply and demand, market equilibrium model can be easily implemented compared to auction and game theoretic spectrum trading models that are much more difficult to realize. ${ }^{1}$ However, sellers and buyers do not directly exchange information among each other. Sellers only communicate with buyers in order to set the equilibrium price that brings supply and demand into balance and enables the exchange to take place, thus resulting in lower communication overhead.

Though, the main wireless resource management problem can be also considered to be a matching problem between resources and users. ${ }^{24}$ In other words, a matching can typically be an assignment between resources and users in the resource management context. Thus, matching theory, born in economics and introduced in $1962,{ }^{25}$ has proved to be a hopeful method for wireless resource allocation, which can defeat the constraints of other mechanisms in terms of complexity, stability, optimality and efficiency. Each user and resource has a maximum allowed number of entities that can be matched with. The ultimate objective of the matching is to match resources and users in an optimal way, taking into account their particular targets. Either side (i.e., resource or user) forms a hierarchical preference table of the other side. The preference results from an objective utility function that measures the satisfaction accomplished by a specific resource-user matching.

The most common classification of matching schemes is established on the maximum allowed number of matched players as follows:

- One-to-one matching (O2OM): Each player of one set can be matched to at most one player of the opposite set. The most popular example is the stable marriage problem in which men and women are matched for marriage.

- One-to-many matching (O2MM): Each player of one set can be matched to multiple players of the opposite set, while in the other set, every player has exactly one match. One known example is the college admissions problem in which a university can recruit multiple students, while a student can be matched to only one university.

- Many-to-many matching (M2MM): Each player within each of the two sets can be matched to more than one player in the opposite set. One related example is the partnerships' creation in peer-to-peer networks.

Matching theory is beneficial for wireless resource management as it introduces suitable solutions in terms of stability and optimality, which precisely indicate different system objectives, and fast, efficient, inherently selforganizing algorithmic implementations. Additionally, it does not demand a centralized controller as the players need not to monitor the preferences of other players. The convergence of the deferred acceptance algorithm ${ }^{25}$ to a stable matching is guaranteed irrespective of the order of play and without any synchronization in time. Besides, matching has been apparently expressed as a simple, efficient, and practical solution framework, which settles the order among self-interested players in the market. Finally, it is applicable to complex networking problems as well, instead of trying to carry out any conventional optimization or game-theoretic approaches. ${ }^{26}$

However, most of the references in literature refer to the user-cell association ${ }^{27}$ and spectrum sharing process in cognitive radio networks, ${ }^{28}$ using only $\mathrm{O} 2 \mathrm{OM}$ and $\mathrm{O} 2 \mathrm{MM}$ models. Furthermore, the matching problem between MNOs and MVNOs in the wireless virtualization field and its efficiency on the spectrum trading market have not been extensively studied yet. A few research works use the concept of matching for the analysis of wireless communication networks, ${ }^{29,30}$ but they just 
analyze the simplified case of the O2MM model. Though, in the real world, MVNOs can cooperate with one or more MNOs. So far, M2MM models have been applied only in research works of different fields such as biology, ${ }^{31}$ economic sciences, ${ }^{32}$ object recognition, ${ }^{33}$ caching. ${ }^{34} \mathrm{~A}$ considerable implementation of matching theory for engineering ${ }^{26}$ is also provided, but it does not clearly investigate the challenges of future wireless systems.

We have already presented the first research work that proposes the more complicated M2MM scheme for spectrum trading in virtualized $5 \mathrm{G}$ networks, ${ }^{35}$ where one MNO can form a partnership with multiple MVNOs and one MVNO can form a partnership with multiple MNOs. We go a step further, and this paper presents an extension of that preliminary evaluation, ${ }^{35}$ thus providing a complete evaluation on the M2MM scheme for spectrum trading in virtualized $5 \mathrm{G}$ networks, since:

- It clarifies how SDN and NFV, as 5G enabler technologies, build the base where new 5G business models will grow. Resource virtualization is set as prerequisite in both research works, however, only in this research work, we obtain a list of such existing implementations.

- It analyzes in more detail how matching theory could be applied in the wireless resource management. In particular, we describe why matching theory outperforms the other applied techniques especially in spectrum trading scenarios.

- It proposes an enhanced version of the utility function. More specifically, this results in matching between MNO and MVNO requests based on the minimum possible amount of unsatisfied requests from both entities, and not based on the close bandwidth and price values.

- It implements more real assumptions in the initialization step of the matching scheme algorithm. In our first research work, ${ }^{35}$ all bandwidth and price requests per entity had the same value. However, this is a special case that does not correspond to reality. In our current research work, the relevant requests differ from each other according to the various requirements or availability offers from the MVNOs and MNOs, respectively. Indeed, these assumptions make this scenario not only real, but also more general and difficult to satisfy both MNOs' and MVNOs' restrictions.

- It examines different evaluation aspects regarding scalability scenarios and the impact of the weighting factors on the efficient resource allocation. First, we increase the number of MVNOs to the greatest number in a European country and check the performance of our matching algorithm in extreme scenarios. Second, we study the influence of the weighting vectors to the performance of the proposed matching scheme. Actually, they enable us to put different emphasis in different parameters, i.e., price, bandwidth, reputation, quality of service, according to the strategy plan.

\section{MATCHING THEORY}

\section{A. Trading Market Nework Model}

MNOs and MVNOs are regarded to be the predominant players in this model:

- MNOs are the traditional mobile network operators, which own (such as infrastructure providers) or control all the resources (i.e., wireless network infrastructure, radio spectrum) required to provide communication services to customers, and also may be interested in selling their resources in the market.

- MVNOs are virtual mobile network operators and play the role of the new market entrants, which do not own wireless network infrastructure and radio spectrum, but they request this kind of resources to satisfy their own customers' needs. More specifically, they lease MNOs' resources and resell them in order to deliver communication services to customers.

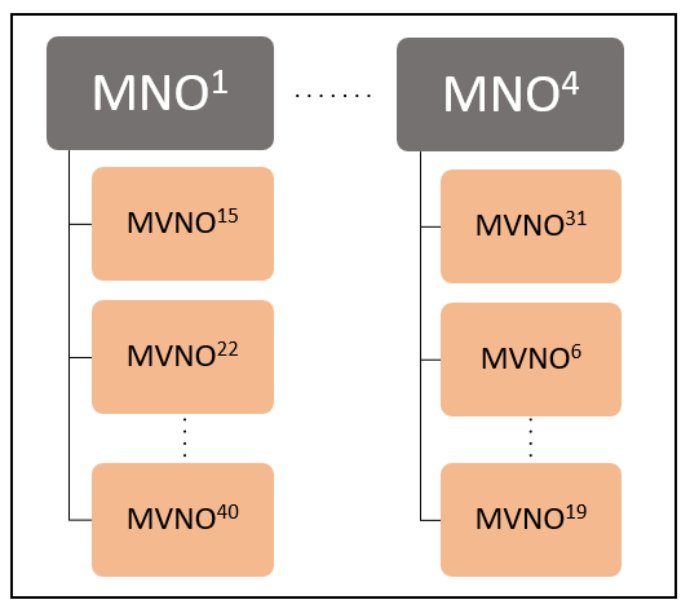

Figure 1. System model under consideration.

The M2MM problem between the set of MNOs and the set of MVNOs is investigated. The system model under consideration is presented in Fig. 1. In this way, $\mathrm{MNO}^{1}$ could possibly form a partnership with $\mathrm{MVNO}^{1}$ and $\mathrm{MVNO}^{2}$, while $\mathrm{MVNO}^{1}$ and $\mathrm{MVNO}^{2}$ could additionally form partnerships with other MNOs, too. Therefore, MNOS are supplied with more economic benefits and MVNOs are given more accessing opportunities. It is considered that the total number of MNOs is $I=4$, while the total number of MVNOs is $J=50$. These correspond to the average numbers in Europe, considering the heterogeneous allocation of MNOs and MVNOs. ${ }^{36}$ 
The initialization step for the trading market between these two entities includes the generation of the market demand and supply. Therefore, all MNOs should announce their available spectrum parts and relevant selling price. In turn, all MVNOs should announce the minimum spectrum part needed and relevant price capable of paying to lease it.

Let $M N O=\{1,2,3, \ldots, I\}$ be the set of $I$ participating MNOs and $M V N O=\{1,2,3, \ldots, J\}$ be the set of $J$ participating MVNOs.

The matching between MNOs and MVNOs is executed using the extension of the deferred acceptance algorithm ${ }^{25}$ for the M2MM case. This means that a MNO can form a partnership with at most $N$ different MVNOs, where $N$ equals to MNO's capacity size, i.e., how many available bandwidth sets each MNO can sell to the market. This also means that a MVNO can form a partnership with at most $M$ different MNOs, where $M$ equals to MVNO's request size, i.e., how many bandwidth sets each MVNO can buy from the market. In our case, where the fixed total number of MNOs and MVNOs is $I=4$ and $J=50$, respectively, the variable values of $N$ and $M$ have been set to 25 and 2, respectively. These values are carefully selected because they result in $I \times N=100$ total available bandwidth sets ready to sell from the MNOs' side and $J \times M=100$ total required bandwidth sets ready to buy from the MVNOs' side. Those variable values of $N$ and $M$ could have also been set to 50 and 4, respectively, thus resulting in total 200 bandwidth sets. Those variable values of $N$ and $M$ could have also been set to 100 and 8 , thus resulting in total 400 bandwidth sets, and so on. However, the combination of values, which yields to the smallest possible number of bandwidth sets (i.e., 100), was selected. Since the supplydemand balance condition is fulfilled, the complete matching of two sets is achievable.

Let Supply $=\{1,2,3, \ldots, N\}$ be the set of $N=25$ requests (i.e., available bandwidth sets to sell) from each MNO and Demand $=\{1, \ldots, M\}$ be the set of $M=2$ requests (i.e., bandwidth sets willing to buy) from each MVNO.

Furthermore, the preference lists of both sets should be strict (i.e., no indifference), if the complete matching is desired to be successful as well. Therefore, both MNOs and MVNOs, set their preference list consisting of the ranking order list of the total opposite set of MVNOs or MNOs, respectively. So, the output of the matching game between MNOs and MVNOs ends up to be a complete and successful matching.

In this work, we propose the formulas of the utility functions $U_{M N O}(I \times N, J \times M)$ and $U_{M V N O}(J \times M, I \times$ $N)$ that will result in the creation of the preference lists of all requests from MNOs and MVNOs, respectively. These utility functions should consist of some specific parameters that each entity considers important to take into account for the creation of its own preference list.

1) Mobile Network Operators: Each MNO decides to advertise its available spectrum to lease and the relevant selling price, i.e., the minimum revenue that a $\mathrm{MNO}$ pursues to gain. Both the bandwidth (BW) and price (PR) level are defined by an integer that follows the uniform distribution in the range [1,5].

$$
\begin{array}{ll}
B W_{M N O}(i, n)=U(1,5) & \forall i, n \in M N O, \text { Supply,resp. } \\
P R_{M N O}(i, n)=U(1,5) & \forall i, n \in M N O, \text { Supply,resp. }
\end{array}
$$

The MNOs' reputation (REP) to the MVNOs and their Quality of Service (QoS) provided to MVNOs' customers could also be employed as two more parameters to characterize MNOs. Both the REP and QoS level are defined by an integer that follows the uniform distribution in the range $[1,3]$.

$$
\begin{array}{ll}
R E P_{M N O}(j, i)=U(1,3) & \forall i, j \in M N O, M V N O, \text { resp. } \\
\operatorname{QOS}_{M N O}(j, i)=U(1,3) & \forall i, j \in M N O, M V N O, \text { resp. }
\end{array}
$$

2) Mobile Virtual Network Operators: Each MVNO decides to advertise its minimum amount of spectrum they need and the price capable of paying to lease it. Both the BW and PR levels are defined by an integer that follows the uniform distribution in the range [1,5].

$$
\begin{gathered}
B W_{M V N O}(j, m)=U(1,5) \\
\forall j, m \in M V N O, \text { Demand,resp. } \\
P R_{M V N O}(j, m)=U(1,5) \\
\forall j, m \in M V N O, \text { Demand,resp. }
\end{gathered}
$$

The MVNOs' reputation to the MNOs could also be employed as one more parameter to characterize MVNOs. The REP level is defined by an integer that follows the uniform distribution in the range $[1,3]$.

$$
R E P_{M V N O}(i, j)=U(1,3) \quad \forall i, j \in M N O, M V N O \text {, resp. }
$$

Note that the BW and PR levels are assumed to be in the range of [1,5], since bandwidth and price are primary and easily countable parameters of the utility function, and also their range must be big enough to reflect the big variety of the relevant requests. Additionally, the REP and QoS levels are assumed to be in the range of $[1,3]$, since reputation and quality of service are secondary and not easily countable parameters of the utility function, and also their range should just reflect a simple satisfaction rate (e.g. bad, medium, good).

So far, there were adequately described the parameters that will selectively compose the utility functions $U_{M N O}(I \times N, J \times M)$ and $U_{M V N O}(J \times M, I \times N)$. However, the utility function should clearly reflect the 
preferences of MNOs and MVNOs. Therefore, a weighting vector could be used in the utility functions' formula and efficiently characterize the significance status of the specific utility functions' parameters. In this way, each entity takes the opportunity to build its own game plan by choosing the most suitable weighting vector. The weighting vectors of the MNOs and MVNOs are shown in detail below. Also, the element values of these weighting vectors are related with each other according to the Equations (1) and (2):

$$
\begin{gathered}
W_{M N O}=\left[W_{M V N O, B W}, W_{M V N O, P R}, W_{M V N O, R E P}\right] \\
W_{M V N O, B W}+W_{M V N O, P R}+W_{M V N O, R E P}=1
\end{gathered}
$$

where $W_{M V N O, B W}$ concerns the MNO's weight for the MVNO's minimum amount of bandwidth demand, $W_{M V N O, P R}$ concerns the MNO's weight for the MVNO's price capable of paying to lease the bandwidth, and $W_{M V N O, R E P}$ concerns the MNO's weight for the MVNO's reputation.

$$
\begin{gathered}
W_{M V N O}=\left[W_{M N O, B W}, W_{M N O, P R}, W_{M N O, Q O S,} W_{M N O, R E P}\right] \\
W_{M N O, B W}+W_{M N O, P R}+W_{M N O, Q O S}+W_{M N O, R E P}=1
\end{gathered}
$$

where $W_{M N O, B W}$ concerns the MVNO's weight for the MNO's bandwidth supply, $W_{M N O, P R}$ concerns the MVNO's weight for the MNO's selling price, $W_{M N O, Q O S}$ concerns the MVNO's weight for the MNO's received quality of service, and $W_{M N O, R E P}$ concerns the MVNO's weight for the MNO's reputation.

\section{B. Matching Schemes}

In our work, in the first stage, we propose the use of the M2MM scheme for the request matching among MNOs and MVNOs. In the second stage, we propose the formula of the utility functions based on which the MNOs and MVNOs will create their own preference lists. Thus, the proposed M2MM algorithm with utility-based preferences is presented in the following.

\section{Many-to-many matching algorithm (M2MM) with utility-based preferences}

\section{Step 1 (Initialization):}

- Each MNO announces its available spectrum to lease, $B W_{M N O}(i, n)$, and relevant selling price, $P R_{M N O}(i, n)$, for all $N$ available sets. Each MNO also maintains a table declaring the reputation level of the MVNOs.

- Each MVNO announces its minimum amount of spectrum needed, $B W_{M V N O}(j, m)$, and price capable of paying to lease it, $P R_{M V N O}(j, m)$, for all
$M$ required sets. Each MVNO also maintains a table declaring the reputation level of the MNOs and the quality of service level granted by the MNOs (in line with the received signal strength).

\section{Step 2 (Preference list):}

- For each $n \in$ Supply request per MNO, a separate preference list for all the MVNOs' requests is constructed according to the utility function $U_{M N O}(I \times N, J \times M)$

- For each $m \in$ Demand request per MVNO, a separate preference list for all the MNOs' requests is constructed according to the utility function $U_{M V N O}(J \times M, I \times N)$.

\section{Step 3 (Matching mechanism):}

- For each $m \in$ Demand request per MVNO, the MVNO checks its preference list and makes a proposal to the highest rated $\mathrm{MNO}$ request.

- Each MNO accepts all the MVNO requests until the MNO's match list size reaches its capacity $N$.

- After this limit, the MNO accepts the $N$ highest rated MVNO requests according to its preference list and rejects the others.

- If any MVNO request gets rejected, then the related MVNO checks its preference list again and makes a proposal to the next highest rated $\mathrm{MNO}$ request, until this MVNO request finds a match.

- The end of the algorithm comes when every MVNO request has matched with a MNO request.

It should be emphasized that MVNOs make the proposal for partnership. This means that MNOs select their match according to their own preference list. This consideration actually implies that MVNOs will probably have better matching, i.e., a matching closer to their own preferences, than MNOs will.

For the evaluation of the utility-based preferences M2MM scheme, it is compared with various schemes:

1. M2MM scheme with random-based preferences, where the preference lists of both MNOs and MVNOs are created in random way. That is, only Step 2 is modified.

2. O2MM scheme with utility-based preferences, where one MNO can associate with multiple MVNOs, but one MVNO can only associate with one MNO. That is, only one of the requests per MVNO gets matched. As a result, probably a large number of $\mathrm{MNO}$ and $\mathrm{MVNO}$ requests remains unmatched.

3. Non-multi-tenancy scheme, where MNOs and MVNOs do not collaborate with each other. Thus, unutilized spectrum remains unavailable because spectrum sharing is not performed. That is, all 
requests from MNOs and MVNOs remain unmatched.

We study the performance of our recommended M2MM scheme with utility-based preferences by establishing a MATLAB simulation model. More specifically, this is a self-made simulation tool that extends the absolutely trusted and stable O2OM algorithm (i.e., deferred acceptance algorithm) to the M2MM algorithm. We have examined a virtualized $5 \mathrm{G}$ network with $I=4$ MNOs of $N=25$ and $J=50$ MVNOs of $M=2$, as introduced in Section III.A. Moreover, if we consider 3 daily spectrum trading interactions on average, then the yearly spectrum trading interactions result in 1080 totally $(3 \times 30$ days $\times$ 12 months $=1080)$. Finally, taking into account that the virtualization concept can be applied (as discussed in Section II), the implementation of our proposed matching algorithm is realistic and can be easily integrated as software (e.g., on a virtual base station). This real implementation capability gives our proposal more power to compete with any other relevant proposals, and to become one of the main operational procedures of the $5 \mathrm{G}$ virtualized business model.

\section{Proposed Utility FunCtion}

The ultimate goal of the M2MM scheme with utilitybased preferences is to achieve the best possible matching that satisfies MNOs and MVNOs at the same time. However, both sides are self-interested. On the one hand, a MNO wishes to get the most suitable MVNOs to host, i.e., the MVNOs that can pay such price that maximizes MNO's revenues. On the other hand, a MVNO wishes to get the most suitable MNOs to be hosted, i.e., the MNOs that can grant such amount of bandwidth that maximizes MVNO's bandwidth reserve to meet its customers' requirements. Therefore, each entity aims to meet its ideal matching pair by maximizing its own utility function. In other terms, the utility function should efficiently represent the preferences of each entity.

\section{A. Utility function formula}

The maximum utility functions of the MNOs and MVNOs are given in the Equations (3) and (4), respectively:

$$
U_{M N O} \max =5 W_{M V N O, B W}+5 W_{M V N O, P R}+3 W_{M V N O, R E P}
$$

$$
\begin{gathered}
U_{M V N O} \max =5 W_{M N O, B W}+5 W_{M N O, P R}+3 W_{M N O, R E P}+ \\
3 W_{M N O, Q O S}
\end{gathered}
$$

where number five corresponds to the biggest grade that the bandwidth or price could be set, and number three corresponds to the biggest grade that reputation or quality of service could be set (cf. Section III.A).

Consequently, the proposed utility function for the MNOs is defined in the Equation (5) as:

$$
\begin{aligned}
& U_{M N O}(i \times n, j \times m) \\
=\mid W_{M V N O, B W} & \left(5+B W_{M V N O}(j, m)-B W_{M N O}(i, n)\right) \\
& +W_{M V N O, P R}\left(5+P R_{M V N O}(j, m)\right. \\
& \left.-P R_{M N O}(i, n)\right) \\
& +W_{M V N O, R E P} R E P_{M V N O}(i, j) \\
& -U_{M N O} \max \mid
\end{aligned}
$$

$\forall i, j, n, m \in M N O, M V N O$, Supply, Demand,resp.

where $U_{M N O}(i, j)$ goes to zero when both differences $B W_{M V N O}(j, m)-B W_{M N O}(i, n)$ and $P R_{M V N O}(j, m)-$ $P R_{M N O}(i, n)$ go to zero, and $R E P_{M V N O}(i, j)$ reaches the peak, i.e., three. This implies that a $\mathrm{MNO}$ request preferably matches with a MVNO request having bandwidth-price sets close to its own ones and the best possible reputation.

Accordingly, the proposed utility function for the MVNOs is defined below in the Equation (6) as:

$$
\begin{aligned}
& U_{M V N O}(j \times m, i \times n) \\
=\mid W_{M N O, B W} & \left(5+B W_{M N O}(i, n)-B W_{M V N O}(j, m)\right) \\
& +W_{M N O, P R}\left(5+P R_{M N O}(i, n)\right. \\
& \left.-P R_{M V N O}(j, m)\right) \\
& +W_{M N O, Q O S} Q O S_{M N O}(j, i) \\
& +W_{M N O, R E P} R E P_{M N O}(j, i) \\
& -U_{M V N O} \max \mid
\end{aligned}
$$

$\forall i, j, n, m \in M N O, M V N O$, Supply, Demand,resp.

where $U_{M V N O}(j, i)$ goes to zero when both differences $B W_{M N O}(i, n)-B W_{M V N O}(j, m)$ and $P R_{M N O}(i . n)-$ $P R_{M V N O}$ (j.m) go to zero, and both $R E P_{M N O}(j, i)$ and $Q O S_{M N O}(j, i)$ reach the peak, i.e., three. This implies that a MVNO request preferably matches with a $\mathrm{MNO}$ request having bandwidth-price sets close to its own ones and the best possible reputation and quality of service.

As a result, the lower the utility function value the better the preference of one entity's request for the matching entity's request.

Finally, it is worth to underline that the utility function value is calculated to set the preference list and evaluate the matching performance:

- The utility function value is computed to set the preference list only for the following schemes: M2MM scheme with utility-based preferences, $\mathrm{O} 2 \mathrm{MM}$ scheme with utility-based preferences. In 
the M2MM scheme with random-based preferences, the preference list is calculated randomly. In the non-multi-tenancy scheme, no preference list is calculated at all.

- The utility function value is computed to evaluate the performance for all schemes. However, we need to clarify that in the cases that there is partial or complete unmatching, i.e., O2MM scheme with utility-based preferences and non-multi-tenancy scheme, respectively, it is assumed that:

$$
\begin{gathered}
B W_{M V N O}(j, m)=P R_{M V N O}(j, m)= \\
R E P_{M V N O}(i, j)=0
\end{gathered}
$$

$\forall i, j, m \in M N O, M V N O$, Demand,resp. in Eq. (5)

$$
\begin{gathered}
B W_{M N O}(i, n)=P R_{M N O}(i, n)=Q O S_{M N O}(j, i) \\
=\operatorname{REP}_{M N O}(j, i)=0 \\
\forall i, j, n \in M N O, M V N O, \text { Supply,resp. in Eq. (6) }
\end{gathered}
$$

\section{B. Same bandwidth and price request values}

Initially, for the sake of less complexity, it is assumed the special case where all bandwidth requests per MNO have the same value and all price requests per MNO have the same value as well. Essentially, our previous research work ${ }^{35}$ was completely based on this assumption for the MNOs, and it is first described in the following:

$$
\begin{gathered}
B W_{M N O}(i, 1)=B W_{M N O}(i, 2)=\cdots=B W_{M N O}(i, n) \\
\forall i, n \in M N O, \text { Supply,resp. } \\
P R_{M N O}(i, 1)=P R_{M N O}(i, 2)=\cdots=P R_{M N O}(i, n) \\
\forall i, n \in M N O, \text { Supply,resp. }
\end{gathered}
$$

Accordingly, our previous research work ${ }^{35}$ was completely based on a similar assumption for the MVNOs, and it is first described in the following:

$$
\begin{gathered}
B W_{M V N O}(j, 1)=B W_{M V N O}(j, 2)=\cdots=B W_{M V N O}(j, m) \\
\forall j, m \in M V N O, \text { Demand,resp. } \\
P R_{M V N O}(j, 1)=P R_{M V N O}(j, 2)=\cdots=P R_{M V N O}(j, m) \\
\forall j, m \in M V N O, \text { Demand,resp. }
\end{gathered}
$$

Obviously, the MNOs put more emphasis on the price parameter, since their target is to increase their earnings. For the MNOs, the bandwidth and the reputation parameter come afterwards. On the contrary, the MVNOs put more emphasis on the bandwidth parameter, since their target is to acquire access to the licensed spectrum. For the MVNOs, the quality of service, price and reputation parameter come afterwards. So, the weighting vectors, assumed in the previous research work, ${ }^{35}$ are the following:

$$
\begin{gathered}
W_{M N O}=[0.3,0.5,0.2] \\
W_{M V N O}=[0.4,0.2,0.3,0.1]
\end{gathered}
$$

Then, the performance of the four different schemes, as mentioned in Section III.B, was evaluated ${ }^{35}$ by calculating the utility function for each one of them. More specifically, the value of the utility function, or just utility, represents the average utility of a request for all requests of one specific entity, for all same type of entities, for 1080 iterations.

Therefore, the average utilities of a MNO and MVNO request were estimated in the four different schemes. ${ }^{35}$ The results showed that the M2MM scheme with utilitybased preferences apparently outperformed over the rest of the schemes. This matching scheme improved the spectrum utilization and also enhanced the welfare of each entity (i.e., revenues for MNOs and bandwidth for MVNOs) involved in the spectrum trading.

Additionally, the average partial utilities of a MNO and MVNO request were estimated in the four different schemes..$^{35}$ It was shown that the matching performance was getting better when the number of parameters in the utility function was growing.

Then, it was assumed ${ }^{35}$ that the $\mathrm{MNO}$ request is satisfied when its matching MVNO request pays same or more money than the announced selling price, otherwise the MNO request is unsatisfied. Accordingly, the MVNO request is satisfied when its matching $\mathrm{MNO}$ request grants same or more spectrum than the announced requesting amount of spectrum, otherwise the MVNO request is unsatisfied. Thus, the satisfaction of a matching pair, which is the union of the aforementioned conditions, is defined as follows:

- If $B W_{M N O} \geq B W_{M V N O}$ and $P R_{M N O} \leq P R_{M V N O}$, then both requests of the matching pair are satisfied.

- If $B W_{M N O}<B W_{M V N O}$ and $P R_{M N O}>P R_{M V N O}$, then both requests of the matching pair are unsatisfied.

- In any other case, one request of the matching pair is satisfied and one is unsatisfied.

Therefore, Fig. 2 and Fig. 3 demonstrate the average number of satisfied and unsatisfied requests from individual entities and matching pairs, respectively, over the M2MM scheme with utility-based preferences and the M2MM scheme with random-based preferences. Both figures appear in our previous research work ${ }^{35}$ and are reproduced in this paper so that the comparison between the different evaluation aspects can be more readable and comprehensible. 
Firstly, in Fig. 2, it can be viewed that the average number of satisfied requests from individual MNOs or MVNOs is bigger than the number of unsatisfied ones in both M2MM schemes, i.e., the M2MM scheme with utility-based preferences (U) and the M2MM scheme with random-based preferences (R). Besides, after comparing these two M2MM schemes, there is not any substantial difference in their performance.

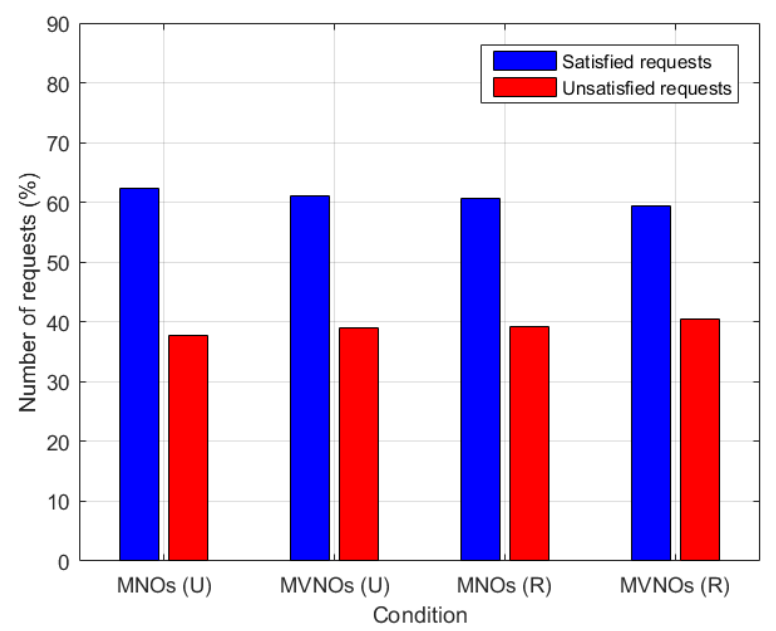

Figure 2. Average number of satisfied and unsatisfied individual requests (same request values). ${ }^{35}$

Secondly, in Fig. 3, it can be noticed that the average number of matching pair requests, that are satisfied, has a considerable superiority in the utility-based preferences M2MM scheme over the random-based preferences M2MM scheme. However, the number of satisfied matching pair requests, i.e., $45 \%$, is less than the number of total unsatisfied ones, i.e., $55 \%$, which drives the need for making severe changes in the proposed utility function in order to effectively defeat this weakness.

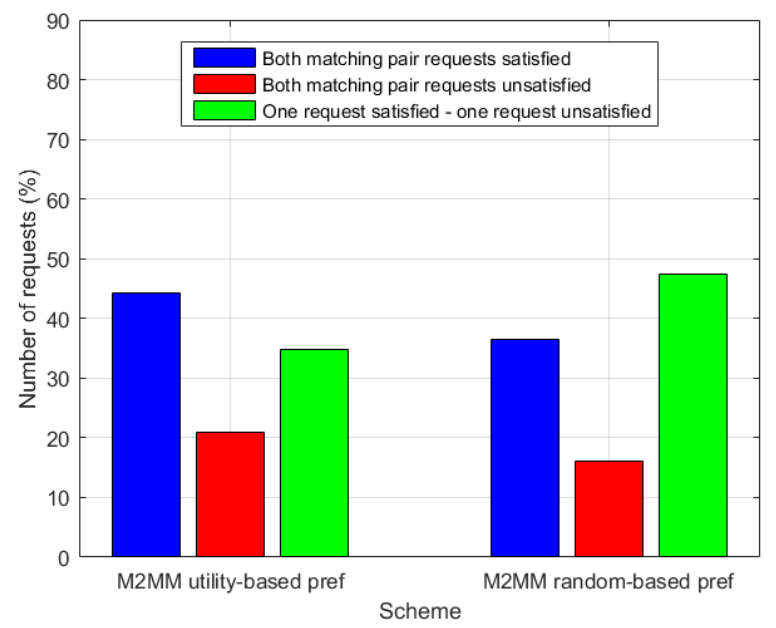

Figure 3. Average number of satisfied and unsatisfied matching pair requests (same request values). ${ }^{35}$

\section{Impact of weighting factors}

In this section, we study the influence of the weighting vectors $W_{M N O}$ and $W_{M V N O}$ to the performance of the proposed utility-based preferences M2MM scheme. ${ }^{35}$ This analysis is considered to be a new contribution of this research work. As mentioned in Section IV.B, the MNOs put more emphasis on the price parameter, since their target is to increase their earnings, while the MVNOs put more emphasis on the bandwidth parameter, since their target is to acquire access to the licensed spectrum. Thus, we focus our results on two main categories:

- First category: Varying the price weight $W_{M V N O, P R}$ of the weighting vector $W_{M N O}$ from 0.1 to 0.9 , by keeping the remaining weights equally distributed. The reference scenario, where $W_{M N O}=[0.3,0.5,0.2]$, and the scenario when all weights have the same value, i.e., $W_{M N O}=[0.33,0.33,0.33]$, are also included. The weighting vector $W_{M V N O}$ remains unchanged.

- Second category: Varying the bandwidth weight $W_{M N O, B W}$ of the weighting vector $W_{M V N O}$ from 0.1 to 0.9 , by keeping the remaining weights equally distributed. The reference scenario, where $W_{M V N O}=[0.4,0.2,0.3,0.1]$, and the scenario when all weights have the same value i.e., $W_{M V N O}=[0.25,0.25,0.25,0.25]$, are also included. The weighting vector $W_{M N O}$ remains unchanged.

At first, for the first category, Fig. 4 depicts the average utilities of a MNO and MVNO request with varying $\mathrm{MNO}$ price weight for our proposed scheme, i.e., M2MM scheme with utility-based preferences. It can be confirmed graphically that the utility of a MVNO request remains almost stable as its utility function is not affected, but the utility of a MNO request normally increases as its main weight (price) increases. After taking into consideration that the lower the utility function value the better the preference of one entity's request for the matching entity's request, as mentioned in Section IV.A, then it seems to be that $W_{M V N O, P R}=0.2$ would probably be the best choice for high utility function performance (for both MNOs and MVNOs). However, Fig. 5 and Fig. 6 , which present the average number of satisfied individual requests and satisfied/unsatisfied matching pair requests, respectively, with varying $\mathrm{MNO}$ price weight for our proposed scheme, prove that $W_{M V N O, P R}=0.2$ would not really be the best choice. In Fig. 5, it is observed that for $W_{M V N O, P R}=0.2$, the difference between the numbers of satisfied MVNO and MNO requests is the largest possible. Only for the reference scenario and $W_{M V N O, P R}=$ 0.5 , there is a balance between the numbers of satisfied 
$\mathrm{MNO}$ and MVNO requests. Note that in the reference scenario, $W_{M V N O, P R}$ is equal to 0.5 , too, as mentioned in Section IV.B. Moreover, in Fig. 6, it is shown that the number of both satisfied matching pair requests can be large for several scenarios, e.g. reference scenario $\left(W_{M V N O, P R}=0.5\right)$ and $W_{M V N O, P R}=0.2,0.3,0.33,0.4,0.7$. Thus, taking into account all these figure results, it is confirmed that our assumption, or reference choice, for $W_{M V N O, P R}=0.5$ does make sense, does achieve fairness between MNO and MVNO entities and does show high performance in terms of both entities' satisfaction. Then, following the same reasoning for varying the MVNO bandwidth weight, we obtain that the best choice is the reference choice with $W_{M N O, B W}=0.4$. We do not include the specific analysis since it follows the same reasoning as for the MNO price weight, and due to space constraints.

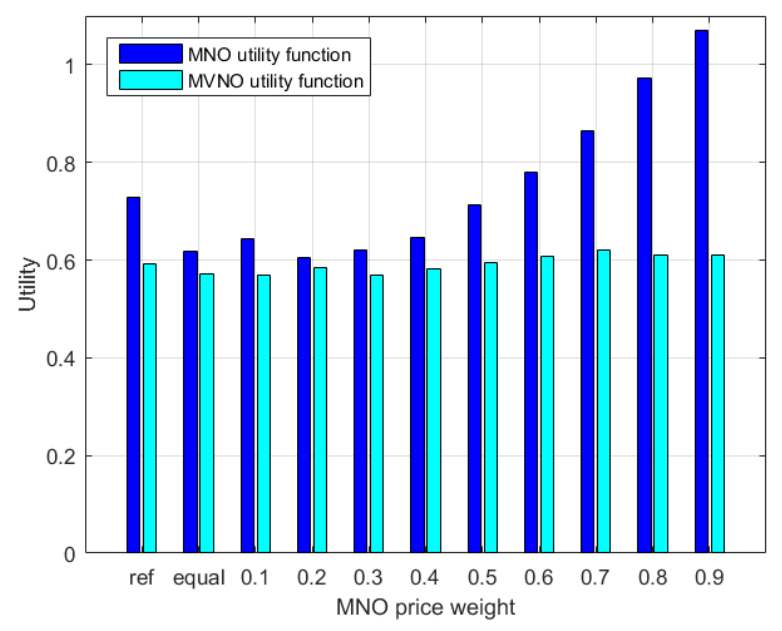

Figure 4. Average utility of a MNO/MVNO request vs. $W_{M V N O, P R}$ for our proposed scheme (same request values).

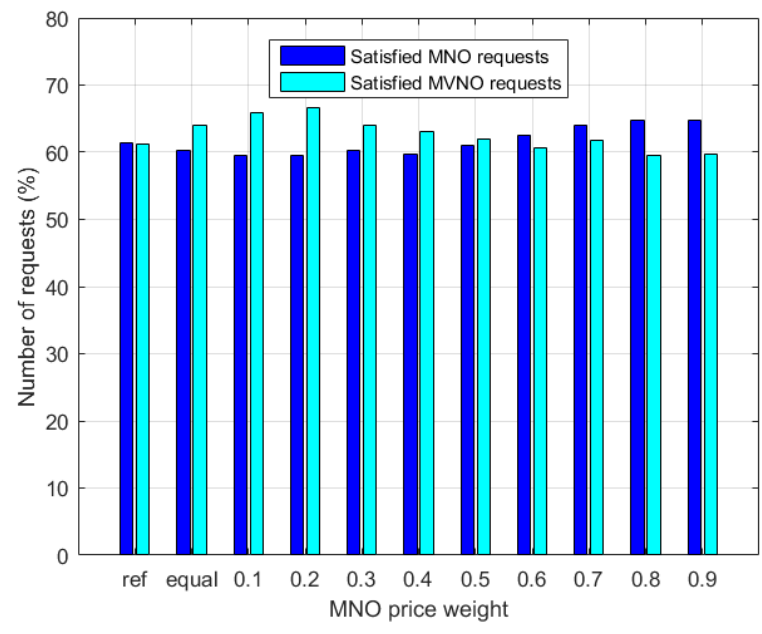

Figure 5. Average number of satisfied individual requests vs. $W_{M V N O, P R}$ for our proposed scheme (same request values).

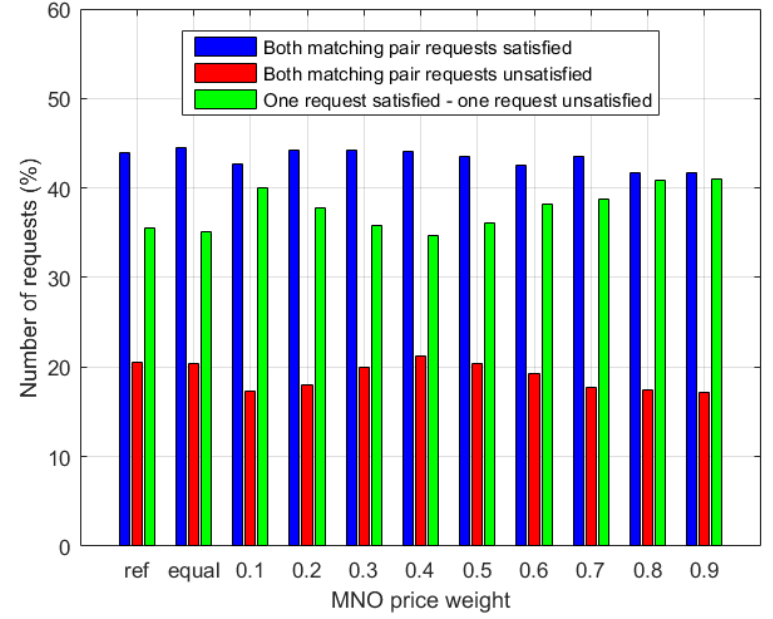

Figure 6. Average number of satisfied/unsatisfied matching pair requests vs. $W_{M V N O, P R}$ for our proposed scheme (same request values).

\section{Scalability evaluation}

The results in our previous research work 35 investigated the performance of the proposed utility-based preferences $\mathrm{M} 2 \mathrm{MM}$ scheme by considering a virtualized $5 \mathrm{G}$ network with $I=4$ MNOs of $N=25$ and $J=50$ MVNOs of $M=2$, as mentioned in Section III.A. Now, in order to have a general view with regard to scalability issues of our proposal, we extend our previous research work's results by increasing the number of MVNOs to 130, which is almost the total number of MVNOs in Germany ${ }^{36}$ and the greatest number in a European country. Thus, in turn, in order to fulfill the supply-demand balance condition, which is mandatory for the complete matching of two sets, as mentioned in Section III.A, we now consider a virtualized $5 \mathrm{G}$ network with $I=4$ MNOs of $N=65$ and $J=130$ MVNOs of $M=2$.

The results showed that the proposed M2MM scheme with utility-based preferences is fully scalable to the number of MVNOs and does not affect the matching efficiency, as its performance remains the same compared with the results in our previous research work. ${ }^{35}$

\section{E. Different bandwidth and price request values}

In our previous research work, ${ }^{35}$ as well as in Section IV.B, the calculation of the utility functions $U_{M N O}(i \times$ $n, j \times m)$ and $U_{M V N O}(j \times m, i \times n)$ was much simplified after the assumptions that all bandwidth requests per entity have the same value and all price requests per entity have the same value as well. That analysis allowed us to validate the utility function and to compare our matching approach with others. However, this is not the general case. In general, the relevant requests differ to each other 
according to the various requirements from the MVNOs or availability offers from the MNOs. Also, it is more difficult to satisfy both MNOs and MVNOs when having different relevant requests, as the variation among the requests is not limited under any condition. The following analysis is considered to be a new contribution of this research work.

At first, the difference between these two cases can be expressed in terms of time complexity:

- In the special case (same request values scenario), in order to set the preference lists, the utility function for all MNOs or MVNOs needs to be calculated $(I \times J)$ times. This happens because all the requests of one MNO are the same and, accordingly, all the requests of one MVNO are the same. As a result, each MNO calculates the utility function $J$ times, totally $(I \times J)$ times for all MNOs ( $I$ MNOs). In the same way, each MVNO calculates the utility function $I$ times, i.e., $(I \times J)$ times for all MVNOs ( $J$ MVNOs).

- In the general case (different requests scenario), in order to set the preference lists, the utility

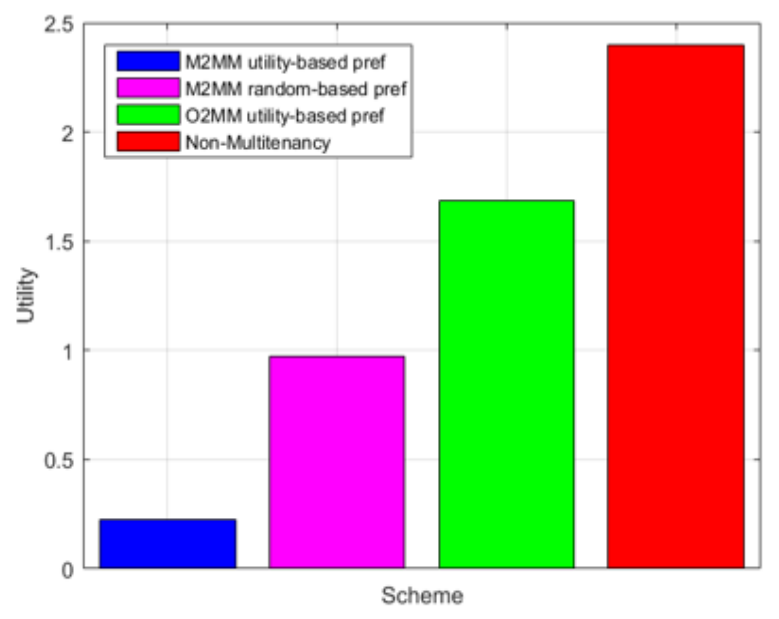

(a) function for all MNOs or MVNOs needs to be calculated $(I \times N \times J \times M)$ times. This happens because all the requests of one $\mathrm{MNO}$ are different ( $N$ requests per $\mathrm{MNO}$ ) and, accordingly, all the requests of one MVNO are different ( $M$ requests per MVNO). As a result, each MNO calculates the utility function $(N \times J \times M)$ times, totally $(I \times N \times J \times M)$ times for all MNOs (I MNOs). In the same way, each MVNO calculates the utility function $(I \times N \times M)$ times, i.e., $(I \times N \times$ $J \times M)$ times for all MVNOs ( $J$ MVNOs).

For example, in this work, given that $I=4, J=50, N=25$ and $M=2$, utility has to be calculated 200 times in the special case and 10.000 times in the general case, i.e., 50 times more. Also, taking into account that we assumed the number of yearly interactions is 1.080 (as mentioned in Section III.B), then the time needed to calculate this general case increases even more. In particular, utility has to be calculated $200 \times 1.080=216.000$ times in the special case and $10.000 \times 1.080=10.800 .000$ times in the general case, which is an extremely demanding task.

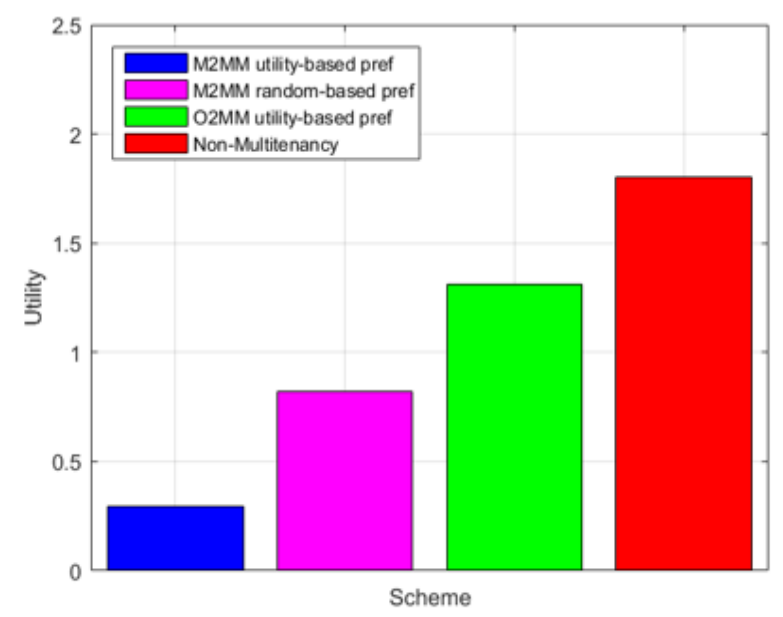

(b)

Figure 7. Average utility of a MNO (a) and MVNO (b) request in four different schemes (different request values).

Secondly, the difference between these two cases can be expressed in terms of performance. Thus, the performance of the four different schemes, as mentioned in Section III.B, is evaluated by calculating the utility function for each one of them, now for the different requests scenario. Then, these utilities are compared with the relevant utilities of the same requests scenario, which was simulated in our previous research work. ${ }^{35}$

More specifically, Fig. 7(a) illustrates the average utility of a MNO request in the four different schemes for the new scenario with different request values. As explained in Section IV.A, the lower the utility function value the better the preference of one entity's request for the matching entity's request. Thus, compared with the results in our previous research work (same request values), ${ }^{35}$ it can be seen that our proposed scheme is the only one among the four schemes that has performance improvement. In fact, the average utility of a MNO request in the utility-based preferences M2MM scheme is 0.75 for the scenario with same request values, and 0.25 for the scenario with different request values.

Additionally, the same behavior is observed when Fig. 7(b), which presents the average utility of a MVNO request in the four different schemes for the new scenario with different request values, is compared with the results in our previous research work (same request values). ${ }^{35}$ 
That means, when the new scenario with different request values is implemented, each MVNO request has more possibilities to find a MNO request closer to its preferences to match, and reversely.

Besides, Fig. 8 and Fig. 9 demonstrate the average number of satisfied and unsatisfied requests from individual entities and matching pairs, respectively, for the new scenario with different request values. In Fig. 8, compared with relevant Fig. 2, it becomes obvious that the average number of satisfied individual requests increases. Accordingly, the average number of unsatisfied individual requests decreases, for the utility-based preferences M2MM scheme (U). In particular, the satisfied MNO requests increase from $62 \%$ to $72 \%$, while the unsatisfied MVNO requests decrease from $38 \%$ to $30 \%$. On the other hand, for the M2MM scheme with random-based preferences (R), the average number of satisfied and unsatisfied individual requests remains the same for both types of requests.

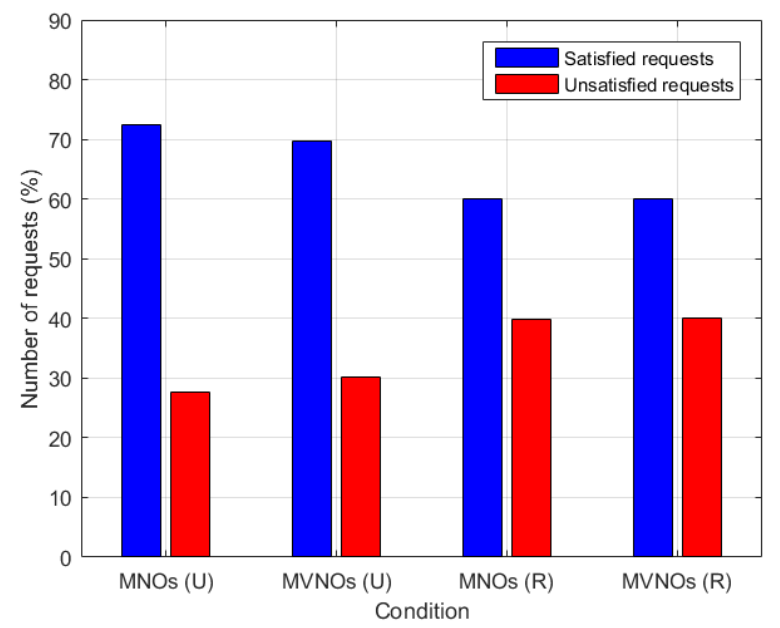

Figure 8. Average number of satisfied and unsatisfied individual requests (different request values).

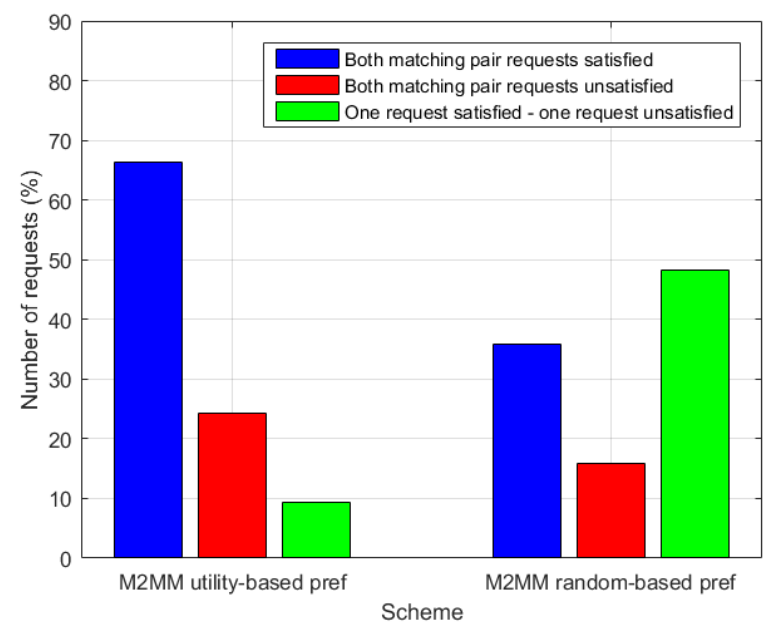

Figure 9. Average number of satisfied and unsatisfied matching pair requests (different request values).
Finally, in Fig. 9, compared with relevant Fig. 3 (same request values), it can be seen that the average number of satisfied matching pair requests is $67 \%$ for the new scenario with different request values, and only $45 \%$ for the previous research work's scenario with same request values, in the M2MM scheme with utility-based preferences. Additionally, the average number of matching pairs with one request satisfied and the other one unsatisfied reduces from $35 \%$ to $9 \%$, but the average number of both unsatisfied matching pair requests increases from $20 \%$ to $24 \%$. Again, for the M2MM scheme with random-based preferences, the performance remains the same for both scenarios.

As a conclusion, it can be stated that our proposed utility-based preferences M2MM scheme contributes to more efficient spectrum trading in the scenario with different request values (general) than in the scenario with same request values (special).

\section{ENHANCED UTILITY FUNCTION}

After taking a more careful look at the proposed utility function of the MNOs and MVNOs in Section IV.A, it is concluded that it encourages the best matching between $\mathrm{MNO}$ and MVNO requests by relying on the minimum possible difference of bandwidth and price values between supply and demand. In other words, it becomes obvious that each entity's request prefers to match with another entity's request having close bandwidth and price values. Additionally, the results of Fig. 3 and Fig. 9, where the average number of both unsatisfied matching pair requests increases from $20 \%$ to $24 \%$, have a negative impact to the utility function's efficiency. These issues imply that the utility function should primarily result in matching between MNO and MVNO requests based on the minimum possible amount of unsatisfied requests from both entities. The following analysis is considered to be a new contribution of this research work.

\section{A. Enhanced utility function formula}

Thus, another version of the utility function should fulfill the aforementioned goal. The enhanced version of utility function for the MNOs is defined below in the Equation (7) as:

$$
\begin{gathered}
U_{M N O}(i \times n, j \times m)=W_{M V N O, B W}(5- \\
\left.\left|B W_{M V N O}(j, m)-B W_{M N O}(i, n)\right|\right)+W_{M V N O, P R}(1+ \\
\left.f\left(P R_{M V N O}(j, m)-P R_{M N O}(i, n)\right)\right)+ \\
W_{M V N O, R E P} R E P_{M V N O}(i, j)
\end{gathered}
$$

$\forall i, j, n, m \in M N O, M V N O$, Supply, Demand,resp. 
where $U_{M N O}(i \times n, j \times m)$ tends to increase when both differences $B W_{M V N O}(j, m)-B W_{M N O}(i, n) \quad$ and $P R_{M V N O}(j, m)-P R_{M N O}(i, n)$ go to zero, and $R E P_{M V N O}(i, j)$ reaches the peak, i.e., three. This implies that a MNO request preferably matches with a MVNO request having bandwidth request close to its own relevant one, price request equal to or larger than its own relevant one and the highest possible reputation. Note that in order to have same range values between the factors (5$\left.\left|B W_{M V N O}(j, m)-B W_{M N O}(i, n)\right|\right) \quad$ and $\quad(1+$ $\left.f\left(P R_{M V N O}(j, m)-P R_{M N O}(i, n)\right)\right)$ in Eq. (7), we follow the mapping in Table 1.

The enhanced version of utility function for the MVNOs is defined below in the Equation (8) as:

$$
\begin{gathered}
U_{M V N O}(j \times m, i \times n)=W_{M N O, B W}(1+ \\
\left.f\left(B W_{M N O}(i, n)-B W_{M V N O}(j, m)\right)\right)+W_{M N O, P R}(5- \\
\left.\left|P R_{M N O}(i, n)-P R_{M V N O}(j, m)\right|\right)+ \\
W_{M N O, Q O S} Q O S_{M N O}(j, i)+W_{M N O, R E P} R E P_{M N O}(j, i)
\end{gathered}
$$

$\forall i, j, n, m \in M N O, M V N O$, Supply, Demand,resp. (8)

where $U_{M V N O}(j \times m, i \times n)$ tends to increase when both differences $B W_{M N O}(i, n)-B W_{M V N O}(j, m)$ and $P R_{M N O}(i, n)-P R_{M V N O}(j, m)$ go to zero, and both $R E P_{M N O}(j, i)$ and $Q O S_{M N O}(j, i)$ reach the peak, i.e., three. This implies that a MVNO request preferably matches with a MNO request having bandwidth request equal or larger than its own relevant one, price request close to its own relevant one, the highest possible reputation and quality of service. Note that in order to have same range values between the factors $(1+$ $\left.f\left(B W_{M N O}(i, n)-B W_{M V N O}(j, m)\right)\right) \quad$ and $\quad(5-$ $\left.\left|P R_{M N O}(i, n)-P R_{M V N O}(j, m)\right|\right)$ in Eq. (8), we follow the mapping in Table 1.

\begin{tabular}{|c|c|c|c|c|c|c|c|c|c|}
\hline $\boldsymbol{x}$ & 4 & 3 & 2 & 1 & 0 & -1 & -2 & -3 & -4 \\
\hline $\boldsymbol{f}(\boldsymbol{x})$ & 4 & 3.5 & 3 & 2.5 & 2 & 1.5 & 1 & 0.5 & 0 \\
\hline
\end{tabular}

Table 1. Mapping values of specific factors

As a result, the higher the utility function value the better the preference of one entity's request for the matching entity's request.

Finally, as clarified in Section IV.A, the utility is calculated to set the preference list and evaluate the matching performance. However, in the cases that there is partial unmatching (i.e., O2MM scheme with utility-based preferences) or complete unmatching (i.e., non-multitenancy scheme), the utility function is expressed differently. It is assumed that the utility functions have the minimum value in these cases:

$$
\begin{aligned}
& U_{M N O}(i \times n, j \times m) \\
& =W_{M V N O, B W}+W_{M V N O, P R} \\
& +W_{M V N O, R E P}=1
\end{aligned}
$$

$\forall i, j, n, m \in M N O, M V N O$, Supply, Demand,resp.

$$
\begin{aligned}
& U_{M V N O}(j \times m, i \times n) \\
& =W_{M N O, B W}+W_{M N O, P R} \\
& +W_{M N O, Q O S}+W_{M N O, R E P}=1
\end{aligned}
$$

$\forall i, j, n, m \in M N O, M V N O$, Supply, Demand,resp.

\section{B. Performance analysis}

Initially, for the enhanced utility function, the weight values, which are expressed in Section IV.B, remain the same as for the original utility function. Besides, a similar weight impact analysis has been done as with the original utility function, and draws the same conclusions, thus validating the reference weight scenario as a valid one.

Then, as analytically described in Section IV.B for the original utility function, for the sake of less complexity, we initially assume the special case where all bandwidth requests per MNO/MVNO have the same value and all price requests per MNO/MVNO have the same value as well. The new average utilities of a MNO and a MVNO request in the four different schemes (enhanced utility function, same request values) are depicted in Fig. 10(a) and Fig. 10(b), respectively. Additionally, as described in Section IV.E, the general case is that the relevant requests differ to each other according to the various requirements from the MVNOs or availability offers from the MNOs. The new average utilities of a MNO and MVNO request in the four different schemes (enhanced utility function, different request values) are illustrated in Fig. 11(a) and Fig. 11(b), respectively.

As explained in Section V.A, for the enhanced utility function, the higher the utility function value the better the preference of one entity's request for the matching entity's request. Therefore, like the original utility function, it can be seen once again that the cooperative schemes (i.e., M2MM and O2MM) show better performance than the non-multi-tenancy scheme. In turn, the utility-based preferences M2MM scheme outperforms all the other schemes. Additionally, like the original utility function, the new average partial utility of a MNO/MVNO request in the four different schemes (enhanced utility function, same request values), as presented at Fig. 12, shows again that the matching performance is getting better when the number of parameters in the utility function is growing. 


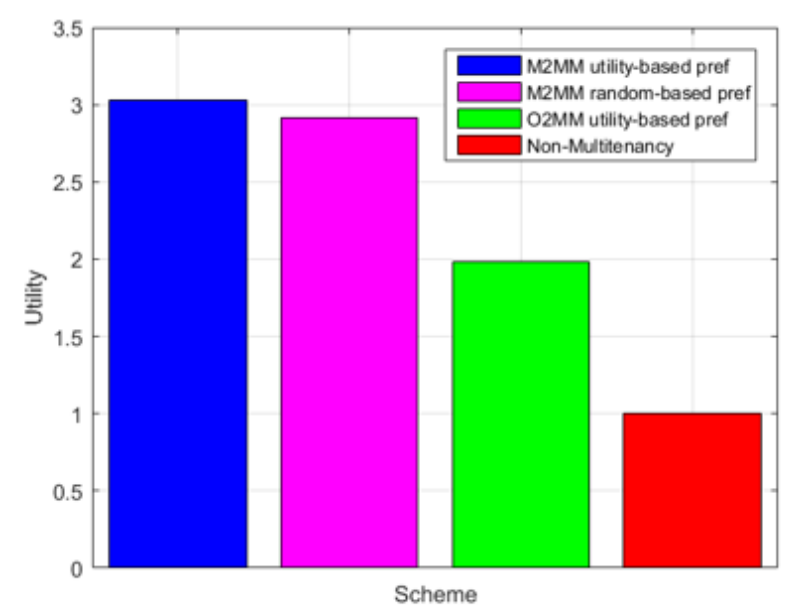

(a)

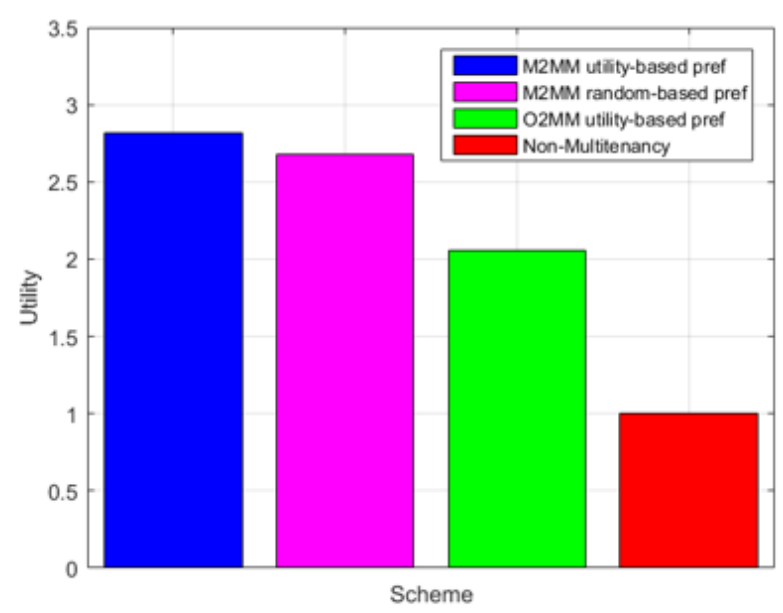

(b)

Figure 10. Average utility of a MNO (a) and MVNO (b) request in four different schemes (enhanced utility function, same request values).

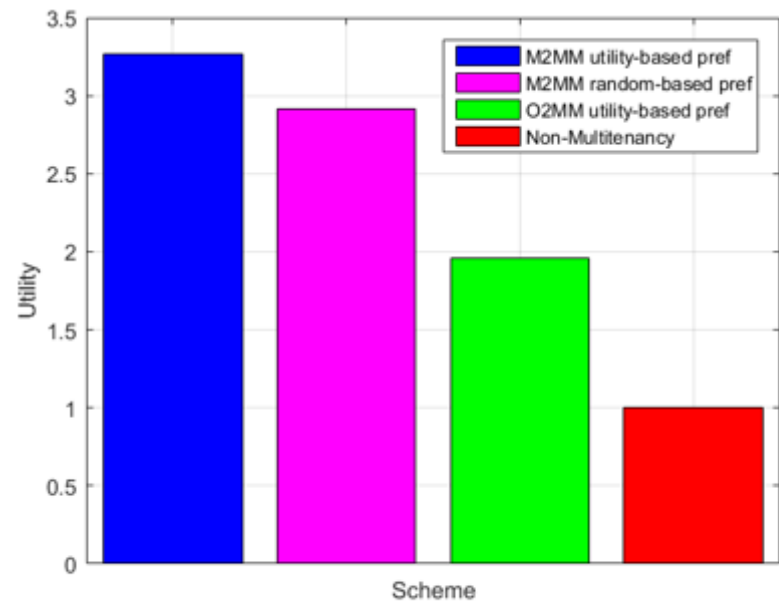

(a)

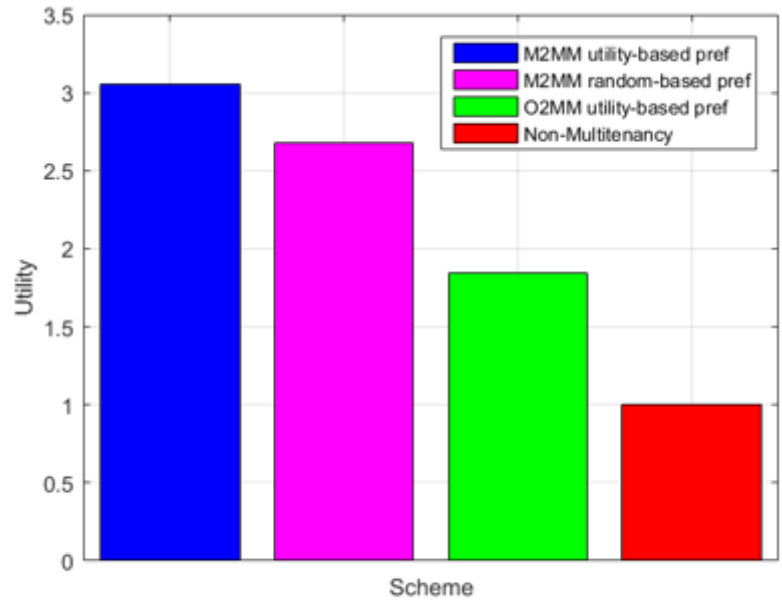

(b)

Figure 11. Average utility of a MNO (a) and MVNO (b) request in four different schemes (enhanced utility function, different request values).

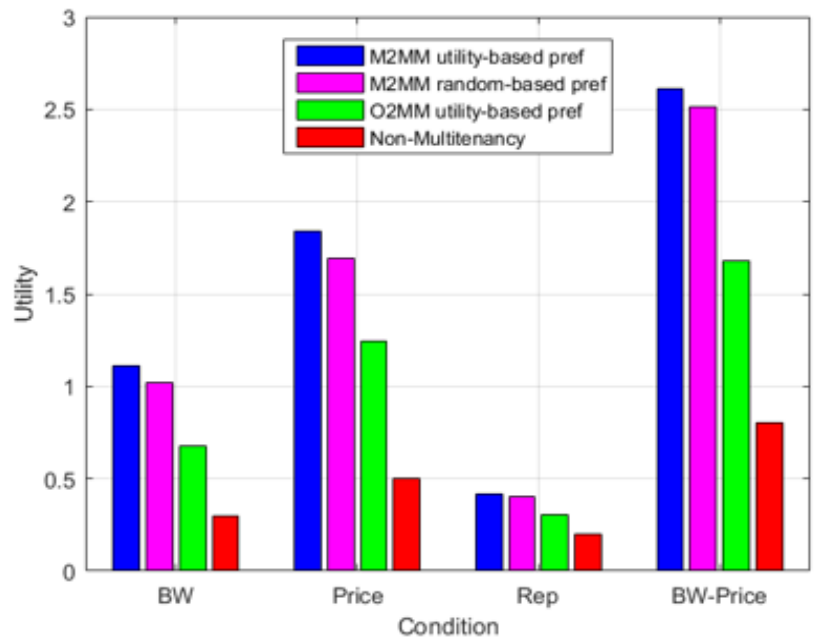

(a)

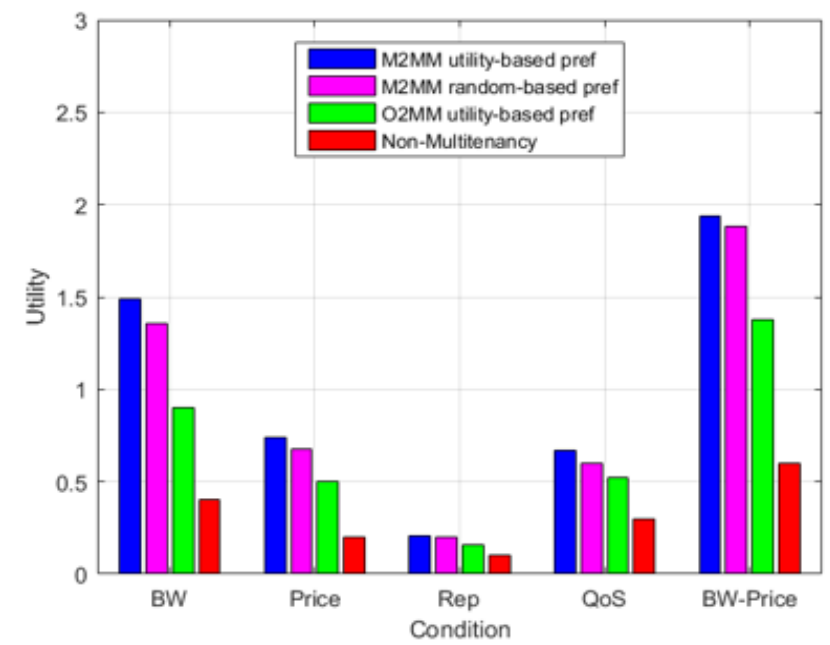

(b)

Figure 12. Average partial utility of a MNO (a) and MVNO (b) request in four different schemes (enhanced utility function, same request values). 
Although Fig. 10 and Fig. 11 show generally that our proposed utility-based preferences M2MM scheme has a superior performance in terms of utility over the other schemes for the enhanced utility function, they do not prove if there is any performance improvement of this new enhanced version over the previous original one. The comparison between the two versions of utility function will be relied on the results related with the average number of satisfied/unsatisfied individual and matching pair requests, which are demonstrated in Fig. 13 and Fig. 14 , respectively.

Firstly, in Fig. 13, compared with relevant Fig. 2 for the scenario of the original utility function with same request values, it can be observed that the new average number of satisfied individual requests increases. Accordingly, the new average number of unsatisfied individual requests decreases for the utility-based preferences M2MM scheme (U). In particular, the new satisfied MNO requests increase from $62 \%$ to $66 \%$, while the new unsatisfied MVNO requests decrease from $38 \%$ to $33 \%$. On the other hand, for the M2MM scheme with random-based preferences (R), the average number of satisfied and unsatisfied individual requests remains the same for both scenarios (original and enhanced utility function).

Secondly, in Fig. 14, compared with relevant Fig. 3 for the case of the original utility function with same request values, it can be seen that the new average number of matching pair requests, that are satisfied, is $45 \%$ for both scenarios (original and enhanced utility function) in the M2MM scheme with utility-based preferences (U). Additionally, the new average number of both matching pair requests, which are unsatisfied, decreases from $20 \%$ to $12 \%$. But the new average number of matching pairs with one request satisfied and the other one unsatisfied slightly increases from $35 \%$ to $42 \%$. Again, for the M2MM scheme with random-based preferences (R), the performance remains the same for both scenarios (original and enhanced utility function).

As a conclusion, it can be stated that our proposed utility-based preferences M2MM scheme and the enhanced utility function with same request values improve the matching performance by radically reducing the number of both matching pair requests that are unsatisfied. Also, the number of both matching pair requests, that are satisfied, remains the same compared with the original utility function with same request values.

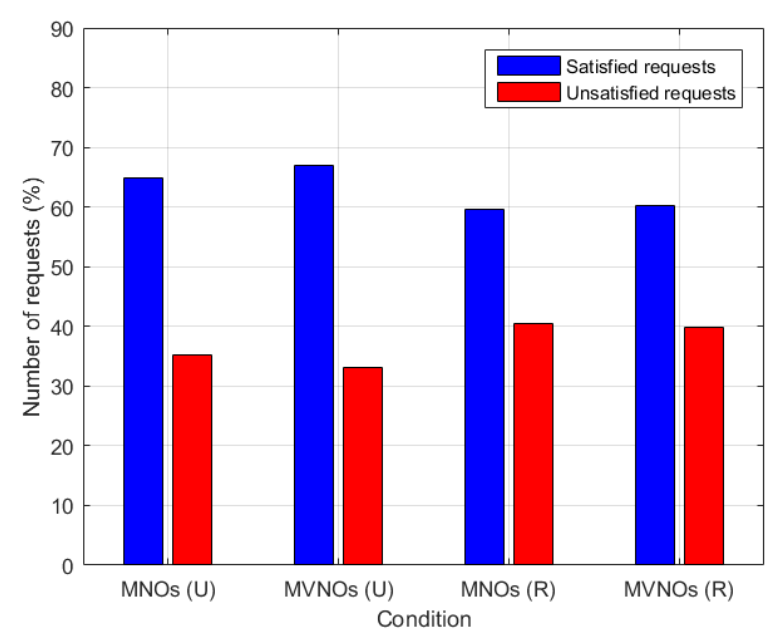

Figure 13. Average number of satisfied and unsatisfied individual requests (enhanced utility function, same request values).

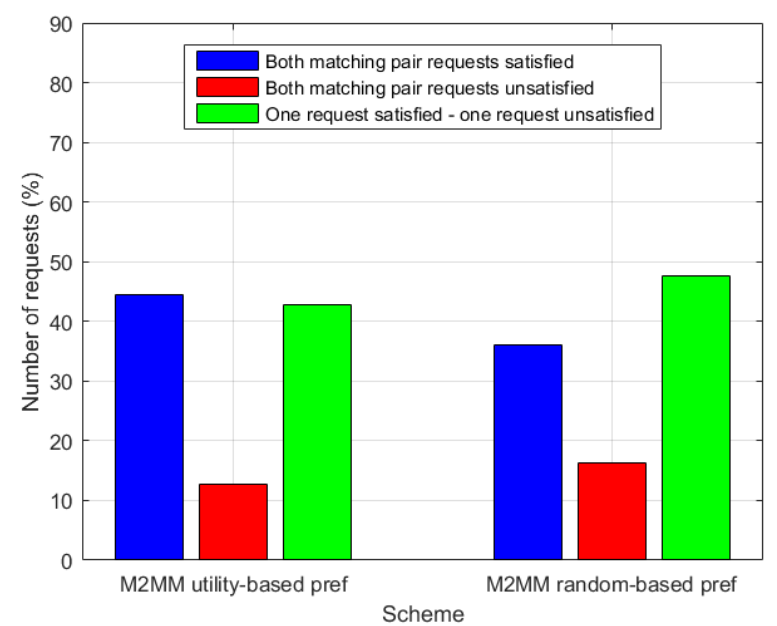

Figure 14. Average number of satisfied and unsatisfied matching pair requests (enhanced utility function, same request values).

Besides, Fig. 15 and Fig. 16 present the average number of satisfied and unsatisfied requests from individual entities and matching pairs, respectively, for the new scenario of the enhanced utility function with different request values.

In Fig. 15, compared with relevant Fig. 13 for the scenario of the enhanced utility function with same request values, we make the same conclusions as for the original utility function, which is presented in Section IV.E. Again, in Fig. 15, compared with Fig. 8 for the original utility function with different request values, it becomes clear that the average number of satisfied individual requests increases and, accordingly, the average number of unsatisfied individual requests decreases for the utilitybased preferences M2MM scheme (U). In particular, the satisfied MNO requests increase from $72 \%$ to $86 \%$, while the unsatisfied MVNO requests decrease from $30 \%$ to $13 \%$. Also, for the M2MM scheme with random-based 
preferences (R), the average number of satisfied and unsatisfied individual requests remains the same for all scenarios.

Then, in Fig. 16, compared with relevant Fig. 14 for the enhanced utility function with same request values, the same conclusions are drawn as for the original utility function, which is described in Section IV.E. Again, in Fig. 16, compared with relevant Fig. 9 for the original utility function with different request values, it can be noticed that the average number of satisfied matching pair requests increases from $67 \%$ to $81 \%$ for the utility-based preferences M2MM scheme (U). Additionally, the average number of both unsatisfied matching pair requests is reduced from $24 \%$ to $9 \%$, while the average number of matching pairs with one request satisfied and the other one unsatisfied increases a bit from $9 \%$ to $10 \%$. Also, for the random-based preferences M2MM scheme (R), the performance remains the same for all scenarios.

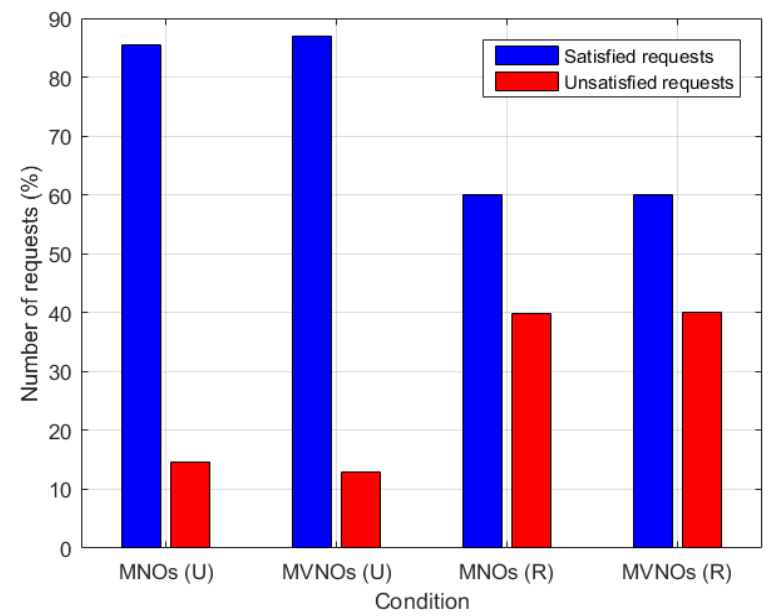

Figure 15. Average number of satisfied and unsatisfied individual requests (enhanced utility function, different request values).

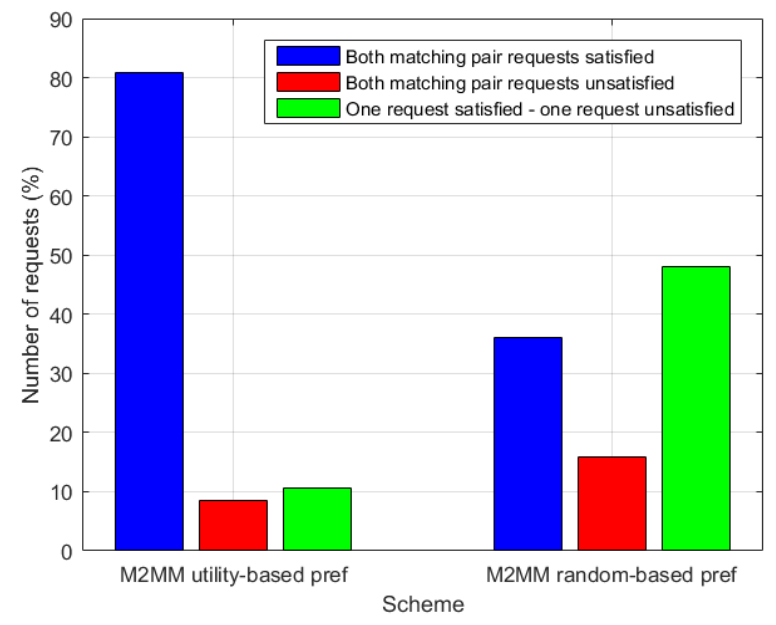

Figure 16. Average number of satisfied and unsatisfied matching pair requests (enhanced utility function, different request values).
Finally, it can be stated that our proposed utility-based preferences M2MM scheme and the enhanced utility function with different request values improve the matching performance by dramatically reducing the number of both unsatisfied matching pair requests. Also, at the same time, they impressively increase the number of both satisfied matching pair requests compared with all other scenarios. Our proposal can match MNO and MVNO requests in such a way that both sides can get the maximum level of satisfaction in terms of matching pair preference.

\section{CONCLUSION AND FUTURE WORK}

This research work has introduced an M2MM scheme with utility-based preferences as a very suitable solution for the spectrum trading problem between the MNOs and the MVNOs of a virtualized $5 \mathrm{G}$ network. Indeed, a detailed description of this matching algorithm and two proposed versions of utility functions (original and enhanced) were provided. Our proposed scheme has been compared with the M2MM scheme with random-based preferences, the O2MM scheme with utility-based preferences and the non-multi-tenancy scheme. It is also evaluated for various system parameters and scenarios to investigate its performance with regard to scalability, strategy plan and generalization. Simulation results indicated that the utility-based preferences M2MM scheme leads to more efficient spectrum utilization and better matching performance for both MNOs and MVNOs as well. Thus, our research work achieved to resolve the spectrum trading problem in a way that both entities are satisfied, as mutually beneficial relations were created between MNOs and MVNOs.

In future, we aim at putting also the end customers (i.e., subscribed users) into the game with MNOs and MVNOs. Thus, we will be able to evaluate their influence to the efficiency of the matching process. Specifically, the MNO is obliged to abstract the physical resources into virtual ones and allocate them to each MVNO. In turn, the MVNO manages the assignment of the slice resources to its end customers accordingly. To this extent, the end customers could actively set their own preference lists for MVNOs or passively affect the requests of MVNOs with their required customized services. This combination of perspectives could give us the opportunity to resolve previously unaddressed issues behind the development of an end-toend matching mechanism, which is appropriate to be served by virtualized $5 \mathrm{G}$ networks. 


\section{ACKNOWLEDGMENTS}

This work has received funding from the European Union Horizon 2020-MCSA-ITN-2015 Innovative Training Networks (ITN) under grant agreement No 675806 (5G-AuRA).

\section{REFERENCES}

1. M.R. Hassan, G.C. Karmakar, G. Kamruzzaman, B. Srinivasan, "Exclusive Use Spectrum Access Trading Models in Cognitive Radio Networks: A Survey", IEEE Communications Surveys and Tutorials, vol. 18, no. 4, pp. 2192-2231, 2017.

2. H. Kim, G. de Veciana, "Leveraging Dynamic Spare Capacity in Wireless Systems to Conserve Mobile Terminals' Energy," IEEE/ACM Trans.on Networking, vol. 18, pp. 802-815, 2010.

3. M. Pan, M. Li, P. Li, Y. Fang, Spectrum Trading in Multi-hop Cognitive Radio Networks. Springer, 2015.

4. E. Hossain, M. Hasan, "5G Cellular: Key Enabling Technologies and Research Challenges," IEEE Instrumentation and Measurement Magazine, vol. 18, no. 3, pp. 11-21, 2015.

5. N. Al-Falahy, O. Y. Alani, "Technologies for $5 \mathrm{G}$ Networks: Challenges and Opportunities", IT Professional, vol. 19, no. 1, pp. 12-20, 2017

6. P. Banelli, S. Buzzi, G. Colavolpe, A. Modenini, F. Rusek, and A. Ugolini, "Modulation Formats and Waveforms for 5G Networks: Who Will Be the Heir of OFDM?," IEEE Signal Process. Mag., vol. 31, no. 11, pp. 80-93, 2014.

7. NGMN Alliance, "Description of Network Slicing Concept," NGMN 5G P1, Jan. 2016.

8. Peter Rost, et al., "Benefits and Challenges of Virtualization in 5G Radio Access Networks," IEEE Communications Magazine, vol. 53, no. 12, pp. 75-82, Dec. 2015.

9. C. Liang and F. R. Yu, "Wireless Network Virtualization: A Survey, some Research Issues and Challenges," IEEE Communications Surveys and Tutorials, vol. 17, no. 1, pp. 358380, 2014.

10. C. Yang, J. Li, M. Guizani, A. Anpalagan, M. Elkashlan, "Advanced Spectrum Sharing in $5 \mathrm{G}$ Cognitive Heterogeneous Networks", IEEE Wireless Communications, vol. 23, no. 2, pp. 94-101, 2016.

11. C.E. Caicedo, M.B.H. Weiss, "The Viability of Spectrum Trading Markets", IEEE Symp. on New Frontiers in Dynamic Spectrum (DySPAN), 2010.

12. K. Zhu, E. Hossain, "Virtualization of $5 \mathrm{G}$ Cellular Networks as a Hierarchical Combinatorial Auction", IEEE Trans. on Mobile Computing, vol. 15, no. 10, pp. 2640-2654, 2015.

13. M. Richart, J. Baliosian, J. Serrat, J. Gorricho, "Resource Slicing in Virtual Wireless Networks: A Survey", IEEE Trans. on Network and Service Management, vol. 13, no. 3, pp. 1-15, 2016.

14. T. Koponen, et al., "Network virtualization in multi-tenant datacenters", in Proc. 11th USENIX Symp. Netw. Syst. Design Implement., pp. 203-216, 2014.

15. S.S. Hong, et al., "Picasso: Flexible RF and Spectrum Slicing", Proc. of the ACM SIGCOMM, 2012.

16. Y. Zaki, et al., "LTE Wireless Virtualization and Spectrum Management", $W M N C, 2011$.

17. C. Liang, F.R. Yu, "Wireless Virtualization for next generation mobile cellular networks", IEEE Wireless Comms, vol. 22, no. 1, pp. 61-69, 2015.

18. X. Wang, et al., "A Collaborative Spectrum-Sharing Framework for LTE Virtualization”, IEEE Conf. on CIC, 2015.
19. Y. Zaki, et al., "LTE mobile network virtualization: Exploiting multiplexing and multi-user diversity gain", Jour. Mobile Networks and Applications, vol. 16, pp. 424-432, 2011.

20. R. Kokku, et al., "NVS: A Substrate for Virtualizing Wireless Resources in Cellular Networks", IEEE/ACM Trans. on Networking, vol. 20, no. 5, pp. 1333-1346, 2012.

21. M. Lopez-Martinez, J.J. Alcaraz, J. Vales-Alonso, J. Garcia-Haro, "Automated Spectrum Trading Mechanisms: Understanding the Big Picture," Wireless Networks, vol. 21, no. 2, pp. 685-708, 2015.

22. B. Liu, H. Tian, "A Bankruptcy Game-based Resource Allocation Approach among Virtual Mobile Operators," IEEE Communications Letters, vol. 17, no. 7, pp. 1420-1423, 2013.

23. M. N. Tehrani, M. Uysal, "Auction Based Spectrum Trading for Cognitive Radio Networks", IEEE communications Letters, vol. 17, no. 6, pp. 1168-1171, 2013.

24. Y. Gu, W. Saad, M. Bennis, M. Debbah, Z. Han, "Matching Theory for Future Wireless Networks: Fundamentals and Applications", IEEE Communications Magazine, vol. 53, no. 5, pp. 52-59, 2015.

25. D. Gale, L. S. Shapley, "College Admissions and the Stability of Marriage," American Mathematical Monthly, vol.69, no.1, pp. 915, 1962.

26. H. Xu, B. Li, "Seen as Stable Marriages", Proc. IEEE INFOCOM, 2011.

27. O. Semiari, W. Saad, S. Valentin, M. Bennis, B. Maham, "Matching Theory for Priority-Based Cell Association in the Downlink of Wireless Small Cell Networks," in Proc. IEEE Int. Conf. on Acoustics, Speech and Signal Processing, pp. 444-448, 2014.

28. S. Bayat, R. Louie, Y. Li, B. Vucetic, "Cognitive Radio Relay Networks with Multiple Primary and Secondary Users: Distributed Stable Matching Algorithms for Spectrum Access," in Proc. IEEE Int. Conf. on Communications, pp. 1-6, 2011.

29. S. Vassaki, M. I. Poulakis, A. D. Panagopoulos, "Spectrum Leasing in Cognitive Radio Networks: A Matching Theory Approach", IEEE Vehicular Technology Conference, 2015.

30. M. M. Gomez, M. B. H. Weiss, G. McHenry, L. Doyle, "Matching Markets for Spectrum Sharing", Telecommunications Policy Research Conference, 2017.

31. M. Baiou, M. Balinski, "Many-to-Many Matching: Stable Polyandrous Polygamy (or Polygamous Polyandry)", Discrete Applied Mathematics, vol.101, no.1-3, pp. 1-12, 2000.

32. A.E. Roth, M.A.O. Sotomayor, "Two-Sided Matching: A Study in Game-Theoretic Modeling and Analysis", Cambridge University Press, 1992.

33. M. F. Demirci, A. Shokoufandeh, Y. Keselman, L. Bretzner, S. Dickinson, "Object Recognition as Many-to-Many Feature Matching”, Int. Journal of Computer Vision, vol.69, no.2, pp. 203-222, 2006.

34. K. Hamidouche, W. Saad, M. Debbah, "Many-to-Many Matching Games for Proactive Social-Caching in Wireless Small Cell Networks", Int. Symp. on Modeling and Optimization in Mobile, Ad Hoc and Wireless Networks, pp. 569-574, 2014.

35. C. Tsirakis, E. Lopez-Aguilera, P. Matzoros, G. Agapiou, D. Varoutas, "Spectrum Trading in Virtualized Mult-Tenant 5G Networks", Int. Symp. on Wireless Communication Systems (ISWCS), 2018.

36. J. Gillet, "Setting the Scene for Future MVNO Growth", GSMA Intelligence Report, 2015. 